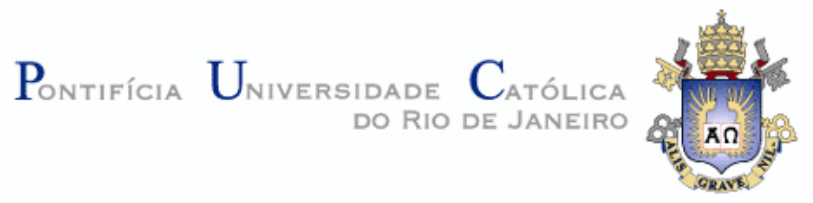

José Carlos Nogueira Siqueira

\begin{abstract}
Medição de petróleo e gás natural: análise crítica dos aspectos de gestão metrológica e de regulação no Brasil
\end{abstract}

Dissertação de Mestrado

Dissertação apresentada como requisito parcial para obtenção do Grau de Mestre pelo Programa de Pós-graduação em Metrologia. Área de Concentração: Metrologia para Qualidade e Inovação da PUC-Rio

Orientador: Prof. Maurício Nogueira Frota, PhD.

Rio de Janeiro

Abril de 2008 


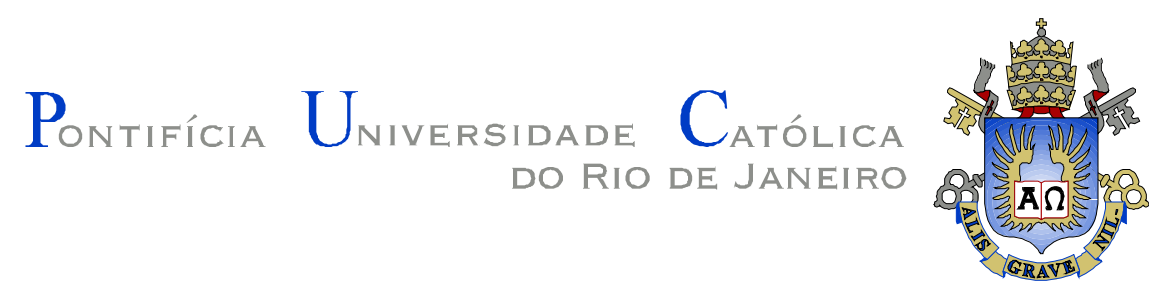

José Carlos Nogueira Siqueira

\section{Medição de petróleo e gás natural: análise crítica dos aspectos de gestão metrológica e de regulação no Brasil}

Dissertação apresentada como requisito parcial para obtenção do grau de Mestre pelo Programa de Pós-Graduação em Metrologia, Qualidade e Inovação do Centro Técnico Científico da Pontifícia Universidade Católica do Rio de Janeiro. Aprovada pela Comissão Examinadora e homologada pela Coordenação Setorial de Pós-Graduação, formalizado pelas respectivas assinaturas.

Comissão Examinadora:

Prof. Dr. Maurício Nogueira Frota

Orientador

Programa de Pós-Graduação em Metrologia (PósMQI)

Pontifícia Universidade Católica do Rio de Janeiro (PUC-Rio)

Profa. Dra. Maria Fátima Ludovico de Almeida

Programa de Pós-Graduação em Metrologia (PósMQI)

Pontifícia Universidade Católica do Rio de Janeiro (PUC-Rio)

Profa. Elisabeth Costa Monteiro

Programa de Pós-Graduação em Metrologia (PósMQI) Pontifícia Universidade Católica do Rio de Janeiro (PUC-Rio)

Prof. Dr. Oswaldo Antunes Pedrosa Jr

Departamento de Engenharia Mecânica Pontifícia Universidade Católica do Rio de Janeiro (PUC-Rio)

Coordenação Setorial de Pós-Graduação:

Prof. José Eugenio Leal

Coordenador Setorial de Pós-Graduação do Centro Técnico Científico (PUC-Rio)

Rio de Janeiro, 15 de abril de 2008. 
Todos os direitos reservados. É proibida a reprodução total ou parcial do trabalho sem autorização da universidade, do autor e do orientador.

\section{José Carlos Nogueira Siqueira}

Graduado em Ciências Econômicas pela Universidade Cândido Mendes (1990), Graduado em Ciências Administrativas pela Universidade Cândido Mendes (1994), Graduado em Direito pela Universidade Estácio de Sá (2001), Pós Graduado pela Pontifícia Universidade Católica do Rio Grande do Sul (2007), Pós-Graduado pela Pontifícia Universidade Católica de Minas Gerais (2007).

Ficha Catalográfica

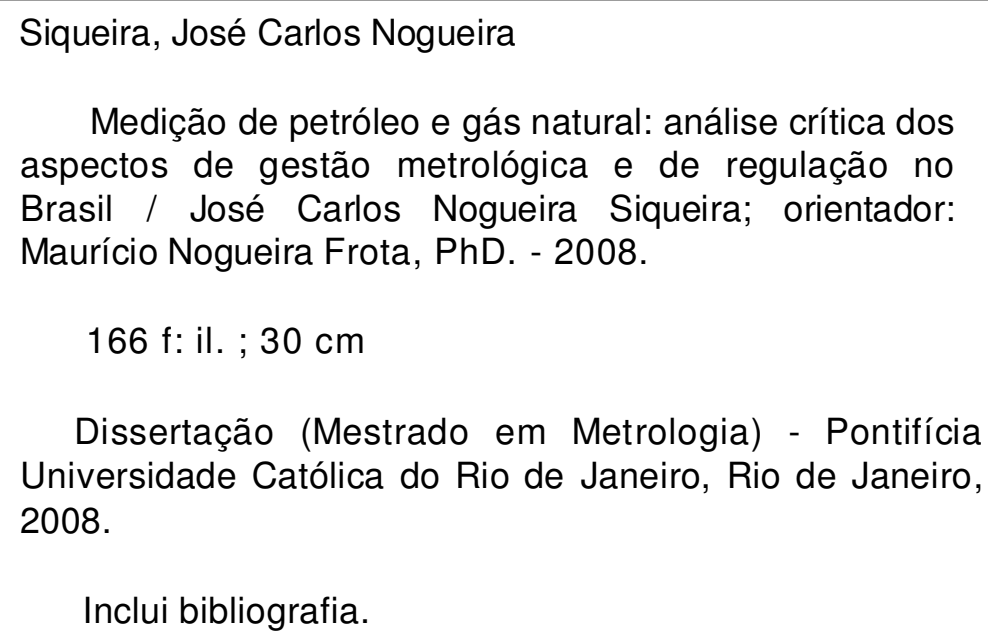

1. Metrologia - Teses. 2. Metrologia. 3. medição de petróleo e gás. 4. regulação. 5. Legislação aplicável à medição. 6. metrologia legal. I. Frota, Mauricio Nogueira. II. Pontifícia Universidade Católica do Rio de Janeiro. Programa de Pós-Graduação em Metrologia para a Qualidade e Inovação. III. Título. 
Dedico este trabalho a minha esposa Gláucia Lilargem e aos meus filhos: Stefanini, Glauber, Jonatha e Estevan Lilargem. 


\section{Agradecimentos}

A Deus em primeiro lugar por mais esta conquista.

Ao Professor Maurício Nogueira Frota, pela sua constante e valorosa orientação e dedicação, não só pelo desenvolvimento deste trabalho, como pelo meu aperfeiçoamento profissional.

À Professora Paula Medeiros Proença de Gouvêa, por toda orientação e ajuda.

Ao Professor Oswaldo A. Pedrosa Jr. Pelo acolhimento e tratamento dispensado a esta causa.

Ao Professor Francisco Mauro Dias, exemplo incólume de vida, pela ajuda incondicional e pelo carinho de sempre.

Ao Eng. José Alberto Pinheiro da Silva Filho, pelo apoio recebido tornando possível esta realização.

A PETROBRAS por mais esta oportunidade, sempre na vanguarda da capacitação tecnológica acreditando na sua força de trabalho e na construção de um país melhor.

A Epifânio Mamani Ticona e Jaime Mamani Ticona pelo competente apoio na edição Latex do trabalho.

À Márcia Ribeiro, secretária do PósMQI, pelo dedicado apoio administrativo.

A PUC-Rio, pelo ambiente acadêmico-científico favorável no desenvolvimento deste trabalho. 


\section{Resumo}

Siqueira, José Carlos Nogueira; Frota, Maurício Nogueira. Medição de petróleo e gás natural: análise crítica dos aspectos de gestão metrológica e de regulação no Brasil. Rio de Janeiro, 2008. 166p. Dissertação de Mestrado - Programa de Pós Graduação em Metrologia. Área de Concentração: Metrologia para Qualidade e Inovação, Pontifícia Universidade Católica do Rio de Janeiro.

Dadas as expectativas de grandes descobertas de petróleo no Brasil (que incluem o pré-sal) a recente legislação que regulamenta a medição de vazão de petróleo e gás natural no país —e que faz intenso uso de infra-estrutura laboratorial- está sendo revista. Â presente pesquisa de mestrado tem por objetivo desenvolver uma análise crítica envolvendo aspectos técnicos e legais do Regulamento Técnico de Medição de Petróleo e Gás Natural (RTM) instituído pela Portaria Conjunta ANP-INMETRO 001/2000. A motivação institucional para o desenvolvimento da pesquisa origina-se do interesse da Petrobrás em colaborar para o avanço do conhecimento relacionado às tecnologias e aos aspectos legais da medição. E, também, da experiência do autor no setor de contratos da Petrobrás cuja problemática da medição da produção de petróleo e gás natural está sendo revista. O trabalho foi desenvolvido no contexto de recentes descobertas de campos gigantes em águas profundas e ultra-profundas. Após o anúncio dessas novas reservas, agentes econômicos têm pressionado a Agência Nacional de Petróleo (ANP) para revisar e reavaliar o Regulamento Técnico de Medição instituído pela Portaria Conjunta ANP-INMETRO 001/2000. O trabalho seguiu os seguintes preceitos metodológicos (i) estudo de legislações aplicáveis à medição de petróleo e gás de países industrializados; (ii) entrevista com especialistas do INMETRO, ANP, Petrobrás (CENPES, divisão de contratos) e com dirigentes técnicos dos principais laboratórios de medição de vazão de petróleo e gás ern operação no país (IPT, CT-PETRO, CT-GÁS, CONAUT, METROVAL, EMERSON) para avaliar pontos críticos da Portaria Conjunta ANP-INMETRO; (iii) análise da consistência e adequação técnica e jurídica da referida Portaria e (iv) identificar novas demandas e necessidades da indústria de petróleo e gás natural no país. Dentre os resultados da pesquisa destacam-se a identificação e a classificação de aspectos técnicos e legais da Portaria ANP-INMETRO 001/2000 que carecem de revisão. Como conclusão, o trabalho sugere a adaptação da legislação vigente para fazer frente a novos desafios impostos ao setor.

\section{Palavras-chave}

Metrologia, medição de petróleo e gás. regulação, legislação aplicável à medição, metrologia legal. 


\section{Abstract}

Siqueira, José Carlos Nogueira; Frota, Maurício Nogueira. Flow measurement of oil and natural gás in Brazil: management and reg-ulation. Rio de Janeiro. 2008. 166p. Dissertation of Master's Degree Program of Pos-Graduation in Metrology. Área of Concentration: Metrology for Quality and Innovation (PÓS-MQI), Pontifical Catholic University of Rio de Janeiro.

Because of the expectation for further massive oil fmdings in Brazil's continen tal platform -including the presalt play-, the existing laws which regnlate the oil and natural gás measurement involving intensive use of laboratory infras-tructure are being reviewed. The objective of this M.Sc.'s research project is to assess legal and technical aspects of the Joint Administrative Rule ANP-INMETRO / 001/2000, specifically the Technical Regulation of Oil and Natural Gás Measurement (RTM).The inotivation of this work emerged frorn the anthors own experience at the legal department of Petrobras dealing with custody transfer contracts where measurements of the production of oiland natural gás play a key economic role. Following the announcements of the ultra-deep giant fields, the work was developed in an economic context where agents are pressing the Brazilian Petroleum Agency (ANP) -the na- tional oil and gás regulator- to review and reassess the applicable Technical Regulation of Measurement (RMO). The project followed four methodolog-ical precepts (i) study of applicable laws to the flow measuremeut of oil and gás in developed countries; (ii) interview with experts of INMETRO, ANP,PETOBRAS (CENPES, division of contracts) and technical managers of themain laboratories involved in flow measurements (IPT, CT-PETRO, CTGAS,CONAUT, METROVAL, EMERSON) to ascertain criticai points in the Joint Administrative Rule ANP-INMETRO; (iii) analysis of consistency of legal and technical aspects of the Administrative Rule and (iv) identify new demands and needs of the oil and gás industry in the country. As the main result, the work identified and characterized technical and legal aspects of the legislation related to oil and gás flow measnrements to help reformulate and update the Administrative Rule, ANP-INMETRO 001/2000. In conclusion, the oil and gás legislation conccrning the Technical Regulation and Measurement should be adapted to new exploration challenges.

\section{Keywords}

1. Metrology. 2. Petroleum and gas flow measurement. 3. Legislation applicable to measurements. 4. Regulation. 5. Legal Metrology. 


\section{SUMÁRIO}

1 Introdução 16

1.1 Objetivos 16

$\begin{array}{lll}1.2 & \text { Metodologia } & 17\end{array}$

$\begin{array}{lll}1.3 & \text { Estrutura } & 17\end{array}$

2 Setor de petróleo e gás natural no Brasil: breve histórico 20

$2.1 \quad$ Fim do monopólio do petróleo 20

2.2 Do monopólio à regulação 21

2.3 O novo regime regulatório 23

2.3.1 O Papel da ANP 23

2.3.2 Portarias da ANP $\quad 25$

2.4 O contexto atual 27

2.4.1 O arcabouço legal; Lei no 9.748/97 e Decreto 2.705/98 27

2.4.2 Participações políticas para mudança da Lei do Petróleo 31

2.4.4 Lei do Gás Natural 21

$3 \quad$ Experiência brasileira na regulação da medição 34

3.1 Regulação 34

3.2 A importância da regulação 36

3.3 Regulação no segmento upstream e downstream 37

3.4 Papel da ANP no cenário de concessões de produção 37

3.5 Atribuições da ANP nas questões de medição da produção 38

3.5.1 Controle da produção de petróleo e gás natural 39

3.5.2 Controle de atividades e investimentos 41

3.5.3 A fiscalização da medição de petróleo e gás natural 41

3.5.4 Integridade dos sistemas operacionais 42

3.5.5 Controle na medição da queima de gás natural 43

3.5.6 Gestão das concessões 43

3.6 Controle e distribuição das participações governamentais 44

3.6.1 Royalities do petróleo e gás natural 45

$\begin{array}{lll}\text { 3.6.2 Metodologia de cálculo } & 47\end{array}$

4 A Experiência internacional na gestão da medição $\quad \mathbf{5 0}$

4.1 A experiência dos Estados Unidos 50

4.1.1 Estrutura institucional $\quad 50$

4.1.2 Regime de outorga $\quad 52$

4.1.3 Regime tarifário 53

4.1.4 Exploração e produção $\quad 54$

4.2 A experiência do Canadá $\quad 55$

4.2.1 Estrutura institucional $\quad 55$

4.2.2 Regime de outorga $\quad 56$

4.2.3 Regime tarifário 56

4.2.4 Exploração e produção 56 
4.3 A experiência da Austrália 58

4.3.1 Estrutura institucional 58

4.3.2 Regime de outorga 59

4.3.3 Regime tarifário 59

4.3.4 Exploração e produção 60

4.4 A experiência do Reino Unido 61

4.4.1 Estrutura institucional $\quad 61$

4.4.2 Regime de outorga $\quad 61$

4.4.3 Regime tarifário $\quad 62$

4.4.4 Exploração e produção 63

4.5 A experiência da Noruega 65

4.5.1 Estrutura institucional 65

$\begin{array}{ll}\text { 4.5.2 Regime tarifário } & 67\end{array}$

$\begin{array}{lll}\text { 4.5.3 Regime de outorga } & 68\end{array}$

4.5.4 Exploração e produção 68

4.6 Regulamentação dos sistemas de medição: estudo comparativo 71

5 Regulamentação técnica da medição de petróleo e gás natural no Brasil $\quad 74$

5.1 Escopo 74

$\begin{array}{lll}5.2 & \text { Abrangência } & 76\end{array}$

5.3 Recursos necessários $\quad 79$

5.3.1 Medição de petróleo 79

5.3.2 Medição de gás 81

5.4 Procedimentos operacionais 83

5.5 Resultados esperados 85

5.5.1 Condiçoes de referência (ou condiçoes base) 86

5.6 Gestão metrológica 86

5.6.1 Recursos Humanos e treinamentos 87

5.6.2 Inspeções e auditorias internas 88

5.6.3 Supervisão de processo e validação de resultados 88

5.6.4 Análise crítica dos resultados 89

5.7 O Papel do INMETRO 89

5.7.1 O Controle metrológico 90

5.7.2 Portaria ํㅜ 64 de 2003

5.8 Rede Brasileira de Calibração 95

5.9 Benefícios da regulamentação do setor petrolífero 96

5.9.1 Pesquisa e Desenvolvimento (P\&D) 96

5.9.2 Fortalecimento da infra-estrutura laboratorial 98

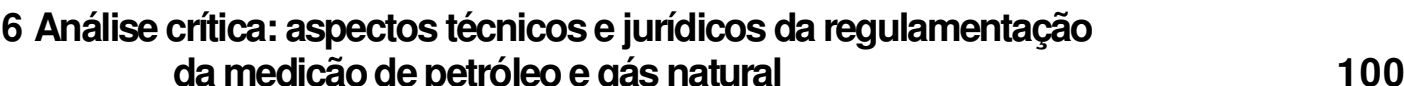

6.1 Adoção de diretrizes para novos cenários de produção 101

6.2 Atualização das referênicas metrológicas 101

6.3 Flexibiliação de sistemas e procedimentos previstos no RTM 102 
6.4 Aprovação de novas tecnologias 103

6.5 Flexibilização da regulamentação: campos marginais e maduros $\quad 104$

6.5.1 O caso particular dos Estados Unidos 107

6.6 Aprovação pela ANP de processos alternativos ao RTM 107

6.7 Visão crítica a legislação: Lei n`9.478/97 108

7 Conclusões e recomendações 109

$\begin{array}{ll}\text { Referências Bibliográficas } & 112\end{array}$

A PORTARIA CONJUNTA N ${ }^{\circ} 001$ ANP/INMETRO (2000) 116

B LEI DO PETRÓLEO (N9.478, DE 6.8.1997) 133

C LEI N ${ }^{\circ}$ 7.990, DE 28.12.1989 148

D DECRETO N².705, DE 3.8.1998 - DOU 4.8.1998

E QUESTÕES NÃO REGULAMENTADAS: REIVINDICAÇÕES 162 


\section{Lista de figuras}

2.1 Mapa das concessões de exploração e produção 30

2.2 Matriz energética nacional 33

3.1 Evolução da produção de petróleo (bep) entre 2000 a $2007 \quad 40$

3.2 Evolução da produção de gás $\left(\mathrm{m}^{3}\right)$ entre 2000 a 2007

3.3 Estrutura atual da distribuição dos royalties 45

3.4 Evolução da arrecadação dos royalties (1998-2007) 46

3.5 Participação especial: evolução da arrecadação (2000-2007) 46

4.1 Tendência de declínio das reservas no Mar do Norte 63

4.2 Tributos recolhidos no Mar do Norte lado britânico 64

4.3 Reservas da Noruega 68

4.4 Evolução da produção da Noruega 69

5.1 Evolução de sistemas de medição adequados 74

5.2 Controle metrológico segundo o INMETRO 92 


\section{Lista de tabelas}

2.1 Principais instrumentos regulatórios lançados pela ANP 26

3.1 Participações governamentais pagas aos beneficiários. 46

3.2 Parcela de 5\% (Lei $n^{\circ} 7.990 / 89$ e Decreto $\left.n^{\circ} 01 / 91\right)$

3.3 Parcela > de 5\% - Lei $n^{\circ} 9.478 / 97$ e Decreto $n^{\circ} 2.705 / 98$

3.4 Pagamento das participações especiais 49

4.1 Tipos de regulamentos e características dos países 50

4.2 Incertezas de medição aceitáveis conforme o CCO 57

4.3 Incertezas dos sistemas de medição conforme o CCO 58

4.4 Incertezas de medição típicas segundo o DTI 65

4.5 Incertezas de medição aceitáveis conforme o NPD 70

4.6 Quadro comparativo regulatório dos países selecionados 71

5.1 Erros máximos na medição de grandezas associadas 80

5.2 Incertezas máximas admissíveis na medição de gás 83

5.3 Recursos para a medição dos volumes 84

5.4 Níveis de erros e incertezas de medição segundo o RTM 85

5.5 Laboratórios nacionais acreditados da RBC 96

E.I Planilha de reivindicações sobre questões não regulamentadas pelo RTM 


\section{Siglas e Abreviações}

$\begin{array}{ll}\text { ABNT } & \text { Associação Brasileira de Normas Técnicas } \\ \text { ACCC } & \text { Australian Competition and Consumer Commission } \\ \text { AGA } & \text { American Gás Association } \\ \text { ANP } & \text { Agência Nacional do Petróleo, Gás Natural e Biocornbustíveis } \\ \text { API } & \text { American Petroleum Institute } \\ \text { ASME } & \text { Sociedade Americana de Engenheiros Mecânicos } \\ \text { ATO } & \text { Gabinete de Impostos Australiano } \\ \text { BERR } & \text { Department for Business, Enterprise and Regulatory Rcforin } \\ \text { BLM } & \text { Bureau of Land Management } \\ \text { BMP } & \text { Boletins Mensais de Produção } \\ \text { CADE } & \text { Conselho Administrativo de Direito Económico } \\ \text { CEFET-RN } & \text { Centro Federal de Educação Tecnológica do Rio Grande do } \\ & \text { Norte } \\ \text { CEFET-SE } & \text { Centro Federal de Educação Tecnológica de Sergipe } \\ \text { CETEC } & \text { Centro Tecnológico de Minas Gerais } \\ \text { C-NOPB } & \text { Canada-Newfoundland offshore Petroleum Board } \\ \text { CNP } & \text { Conselho Nacional do Petróleo } \\ \text { CNPE } & \text { Conselho Nacional de Política Energética } \\ \text { C-NSOPB } & \text { Canada-Nova Scotia offshore Petroleum Board } \\ \text { CONMETRO } & \text { Conselho Nacional de Metrologia, Normalização e Qualidade } \\ & \text { Industrial } \\ \text { CPP } & \text { Contratos de Produção Partilhada } \\ \text { CRF } & \text { Código de Regulamentação Federal } \\ \text { CT-GAS } & \text { Centro de Tecnologia do Gás } \\ \text { CT-PETRO } & \text { Fundo Setorial do Petróleo e Gás Natural } \\ \text { DMPR } & \text { Departamento de Minerais e Recursos Petrolíferos } \\ \text { DOE } & \text { Department of Energy } \\ \text { DTI } & \text { Department of Trade \&; Industry } \\ \text { E\&P } & \text { Exploração e Produção } \\ \text { EERE } & \text { Energy Efficiency and Renewable Energy } \\ \text { EIA } & \text { Energy Information Administration } \\ \text { FAURGS } & \text { Fundação Universidade do Rio Grande do Sul } \\ & \end{array}$




$\begin{array}{ll}\text { FERC } & \text { Federal Energy Regulatory Conimission } \\ \text { GLO } & \text { General Larid Office } \\ \text { IBP } & \text { Instituto Brasileiro do Petróleo } \\ \text { INMETRO } & \text { Instituto Nacional de Metrologia, Normalização e Qualidade } \\ & \text { Industrial } \\ & \\ \text { INPM } & \text { Instituto Nacional de Pesquisas e Medidas } \\ \text { IPT } & \text { Instituto de Pesquisas Tecnológicas de São Paulo } \\ \text { IQM } & \text { Instituto de Qualidade e Metrologia } \\ \text { ISO } & \text { International Organization for Standardization } \\ \text { ITUC } & \text { Instituto Tecnológico da Pontifícia Universidade Católica do } \\ & \text { Rio de Janeiro } \\ & \\ \text { MCT } & \text { Ministério da Ciência e Tecnologia } \\ \text { MME } & \text { Ministério das Minas e Energia } \\ \text { MMS } & \text { Mineral Management Service } \\ \text { NCC } & \text { National Competition Council } \\ \text { NEB } & \text { National Energy Boar } \\ \text { NFP } & \text { Núcleo de Fiscalização da Medição de Petróleo e Gás Natural } \\ \text { NGPAC } & \text { National Pipeline Advisory Committe } \\ \text { NPD } & \text { Norwegian Petroleum Directorate } \\ \text { NSOGM } & \text { Sociedade Noruega de Medição de Petróleo e Gás } \\ \text { OCS } & \text { Outer Continental Shelf } \\ \text { OCSLA } & \text { Outer Continental Shelf Lands Act } \\ \text { OIML } & \text { International Organization of Legal Metrology } \\ \text { ONM } & \text { Organismos Nacionais de Normalização } \\ \text { OTO } & \text { Gabinete de Impostos Petrolíferos } \\ \text { PAP } & \text { Programas Anuais de Produção } \\ \text { PAT } & \text { Programas Anuais de Trabalho e Orçamento } \\ \text { PCS } & \text { Poder Calorífico Superior } \\ \text { PD } & \text { Plano de Desenvolvimento } \\ \text { PE } & \text { Participações Especiais } \\ \text { PEN } & \text { Política Energética Nacional } \\ \text { PETROBRAS } & \text { Petróleo Brasileiro S.A. } \\ \text { PIB } & \text { Produto Interno Bruto } \\ \text { PND } & \text { Programa Nacional de Desestatização } \\ \text { POAG } & \text { Programa de ajuste para a Redução da Queima de Gás Natural } \\ \text { PosMQI } & \text { Programa de Pós-Graduação em Metrologia (PUC-Rio) } \\ \text { PRT } & \text { Taxa de Receita do Petróleo } \\ \text { PUC-Rio } & \text { Pontifícia Universidade Católica do Rio de Janeiro } \\ & \\ \text { ITM } & \\ & \end{array}$


RBC Rede Brasileira de Calibração

RBMLQ-I Rede Brasileira de Metrologia Legal e Qualidade - InMetro

RTM Regulamento Técnico de Medição de Petróleo e Gás

Natural

SBN Sistema Brasileiro de Normalização

SDE Secretaria de Direito Econômico

SDFI State's Direct Financial Interest

SDP Superintendência de Desenvolvimento e Produção

SINMETRO Sistema Nacional de Metrologia, Normalização e Qualidade Industrial

SPL Superintendência de Promoção de Licitações

SPT Imposto Especial de Petróleo

STN Secretaria do Tesouro Nacional

UFBA Universidade Federal da Bahia

UFES Universidade Federal do Espírito Santo

UFRN Universidade Federal do Rio Grande do Norte

UFSC Universidade Federal de Santa Catarina

UIWP Upstream Issues Working Group

UNIFACS Universidade de Salvador

USGS United States Geological Survey

VIM Vocabulário Internacional de Termos Fundamentais e Gerais de Metrologia

WA Austrália Ocidental 